\title{
SEM of seed coat surface and SDS-PAGE of seed protein criteria in certain taxa of the Primulaceae
}

\author{
Abed-El-Moniem I. Aboel-Atta \\ Maged M. Abou-El-Enain \\ and \\ Mohamed H. Loutfy \\ Biological Sciences and Geology Department, Faculty of Education, \\ Ain Shams University, Roxy (Heliopolis), P.C.11341, Cairo, Egypt.
}

\begin{abstract}
Aboel-Atta, A. I.; Abou-El-Enain M. M. \& Loutfy M. H. 2000. SEM of seed coat surface and SDS-PAGE of seed protein criteria in certain taxa of the Primulaceae. Taeckholmia 20(1): 95-111.

SEM of seed coat surface and SDS-PAGE of seed protein criteria were used to re-assess the taxonomic relationships between 12 species belonging to three genera of Primulaceae, viz. Cyclamen L. (one species), Lysimachia L. (six species) and Primula L. (five species). The data obtained, in addition to other characters available in the literature, were analyzed by the NTSYS-pc. Program package using the UPGMA clustering method. A considerable divergence was evident between Cyclamen and each of Lysimachia and Primula. A close relationship was recorded between each of Lysimachia lichiangensis Thunb. \& L. verticillata Bieb; L. ephemerum (Gray) Hbd.\& L. punctata L.; Primula burmanica Fern.\& P. japonica Gray; Primula elatior (L.) Hill \& $P$. veris L. The variations in SDS-PAGE of seed protein profiles were compatible to some extent with the morphological variations exhibited in these taxa. The validity of using the seed coat microsculpture and seed protein electrophoretic criteria as taxonomic evidence in the Primulaceae was referred to.
\end{abstract}

Key words: Cyclamen - Lysimachia - Primula - Primulaceae- SDS-PAGE - SEM

\section{Introduction}

The Primulaceae is a cosmopolitan family widely distributed throughout the cold and tropical zones with a center of diversity in the North temperate regions (Yurtsev et al., 1979; Watson \& Dallwitz, 1999). The family comprises about 22 genera and 825 species, many of which have economic important uses in medicine and ornamentation (Watt \& Breyer-Brandwijk, 1962; Porsild \& Cody, 1980; Mabberley, 1997). The taxonomic relationships within the family have been mainly delimited by the vegetative and floral characters (Pax,1889; Pax \& Knuth, 1905; Ludi, 1927; Pobedimova, 1952; Schwarz,1955; Schwarz \& Lepper, 1964 and Anderberg et al, 2000), anatomical structure (Nishino, 1983; Beyazoglu, 1989), cytological characters (Jorgenson et al., 1958; Sarkar, 1973\&1988), pollen morphology (Spanowsky, 1962) and molecular criteria (Anderberg \& Kallersjo, 1998 and Kallersjo et al, 2000) However, neither the seed coat surface nor seed protein electrophoresis criteria have been used to discuss such relationships in the Primulaceae.

SEM of seed coat surface is a useful technique in the identification and classification of various taxa (Barthlott, 1981; Boesewinkel \& Bouman, 1984). Seed protein banding patterns as revealed by polyacrylamide gel electrophoresis in the presence of Sodium dodecyl sulfate (SDS-PAGE) have provided a valid source of taxonomic 
evidence for addressing the relationships at the different taxonomic levels (Ladizinsky and Hymowitz, 1979; Badr et al., 1998).Variations in SDS-PAGE of seed protein profiles have successfully been used to differentiate between species in a number of genera e.g. Lathyrus (Badr et al., 2000) and families e.g. Solanaceae (Khalifa et al., 1998).

The present work deals with using the SEM of seed coat surface and SDS-PAGE seed protein criteria to provide more information about the taxonomic relationships of 12 species of three genera representing three tribes of the Primulaceae (sensu Pax 1889) i.e Cyclamineae, Lysimachieae and Primuleae. In addition, the available literature was consulted as regards the morphological characters (Bailey, 1949; Mabberley, 1997; Watson \& Dallwitz, 1999), the anatomical characters (Nishino, 1983), the phytochemical characters (Harborne, 1968; Kelso, 1991), the cytological characters (Fedorov, 1969; Goldblatt, 1981\&1988) as well as the geographic distribution of the studied taxa (Valentine, 1972 and Bailey \& Bailey,1976).

\section{Materials and Methods}

The source, origin, diploid and haploid chromosome number of the examined materials are given in Table 1. Voucher specimens are kept at the Department of Biological Sciences, Faculty of Education, Ain Shams University. For the study of seed coat surface, seeds (three seeds for each taxon) were mounted in colloidal silver on copper stubs, coated with a thin layer of gold in Polaron E 5000 and photographed by a JEOL-Scanning Microscope at a magnification of 750 at the Central Lab. of the Faculty of Agriculture, Alexandria University. The terminology of Barthlott (1981), Boesewinkel \& Bouman (1984) and Stearn (1992) was followed to describe the characteristics of the seed coat surfaces.

To extract seed proteins $0.5 \mathrm{~g}$ of mature seeds were mixed with an equal weight of pure, clean, sterile fine sand, powdered using a mortar and pestle and homogenized with $0.2 \mathrm{M}$ Tris- $\mathrm{HCl}$ buffer, $\mathrm{pH}=8$ for $1 \mathrm{~h}$ at $4{ }^{\circ} \mathrm{C}$. The extract was centrifuged at 12000 rpm for $10 \mathrm{~min}$. The supernatant (protein extract) was transferred to new tubes and immediately used for electrophoresis or kept at $-20{ }^{\circ} \mathrm{C}$ until use. For electrophoresis, $40 \mu \mathrm{l}$ of the extract were mixed with an equal volume of a sample buffer $(0.125 \mathrm{M}$ Tris- $\mathrm{HCl}, \mathrm{pH}$ 6.8, $2 \%$ SDS, $10 \%$ sucrose, $0.5 \% \beta$-mercaptoethanol and $0.1 \%$ bromophenol blue as a tracking dye), denatured by boiling for $5 \mathrm{~min}$ in a water bath and cooled. Then, $20 \mu \mathrm{l}$ of this mixture were loaded in $12.6 \%$ slab gel, which was prepared as described by Laemmeli (1970). Electrophoresis was carried out in Tris-Glycine buffer $(\mathrm{pH}=8.3)$ at 4 ${ }^{\circ} \mathrm{C}$ and 125 volt for $2 \mathrm{~h}$ using a Pharmacia low-molecular weight protein mixture as standard marker. Gel was then stained in $0.1 \%$ Comassie Brilliant Blue R-250 for $1 \mathrm{~h}$, destained and photographed while wet and stored for subsequent examination. Total bands in the produced electropherogram were scored and their molecular weights were calculated using the standard protein marker.

For creating a data matrix for numerical analysis of results, the recorded seed protein and SEM of seed coat characters for the taxa studied; as well as the other characters compiled from the literature were scored and coded as shown in Table 2. The relationship between the taxa studied was measured by calculating their average taxonomic distance (dissimilarity) and presented as phenograms based on analysis for each data set separately and for two or more sets in combination (Fig. 3). These analyses 
were performed using the NTSYS program package for IBM-pc as described by Rohlf (1993). In the computer analysis, the taxa are numbered as indicated in Table 1.

\section{Results and Discussion}

For the studied taxa, the SEM micrographs of the seed coat surface are shown in Fig. 1, and their seed protein banding patterns are shown in Fig. 2. A summary of the produced characters recorded in the present study (10 for SEM and 20 for SDS-PAGE) in addition to the compiled data (53 morphological, five phytochemical, two cytological and ten cases of the geographical distribution) and their codes is given in Table 2. The phenograms illustrating the relationships between the studied taxa; based on the analyses of the abovementioned data sets; using the UPGMA clustering method are presented in Fig. 3: A-D.

The description of seed coat scan of the studied taxa is summerized as follows: -

1. Cyclamen persicum Miller Spermoderm reticulate to ribbed, epidermal cells polymorphic. Anticlinal walls slightly wavy, raised and of variable thickness. Periclinal walls appear more or less granular and concave.

2. Lysimachia ciliata $\mathbf{L}$. Spermoderm reticulate to ruminate, epidermal cells polymorphic. Anticlinal walls wavy, very thick and highly raised. Periclinal walls illdefined.

3. L. ephemerum (Gray) Hbd. Spermoderm reticulate, epidermal cells polymorphic. Anticlinal walls slightly wavy, thick and highly raised. Secondary sculpture appear as dense striations running parallel to each other, overlapping the walls and extending into the lumen of the cells. Periclinal walls concave and striated.

4. L. lichiangensis Thunb. Spermoderm reticulate. Epidermal cells polymorphic. Anticlinal walls wavy, thick, slightly raised. Periclinal walls slightly concave, with few tubercules.

5. L. punctata L. Similar to L. ephemerum (Gray) Hbd.

6. L. verticillata Bieb. Similar to $L$. ciliata except that few papillae like structures or tubercules are seen protruding from some periclinal walls.

7. L. vulgaris L. Spermoderm reticulate to favulariate. Epidermal cells polymorphic. Anticlinal walls slightly wavy, highly raised and striated.

8. Primula auricula L. Similar to Cyclamen persicum except that the anticlinal walls are slightly striated. Periclinal walls are slightly striated and concave.

9. P. burmanica Fern. Spermoderm reticulate to scalariform. Epidermal cells polymorphic. Cells generally large in size. Anticlinal walls smooth, raised, and of varying thickness. More or less spherical masses are seen located on parts of the anticlinal walls. Periclinal walls slightly concave with secondary sculpture taking the form of a fine network.

10. P. elatior (L.) Hill Spermoderm reticulate to sulcate. Epidermal cells polymorphic. Anticlinal walls very thick, highly raised and striated. Periclinal walls ill defined. 
A. I. Aboel-Atta, M. M. Abou-El-Enain \& M. H. Loutfy

Table (1): Source, origin, diploid and haploid chromosome numbers of the taxa studied

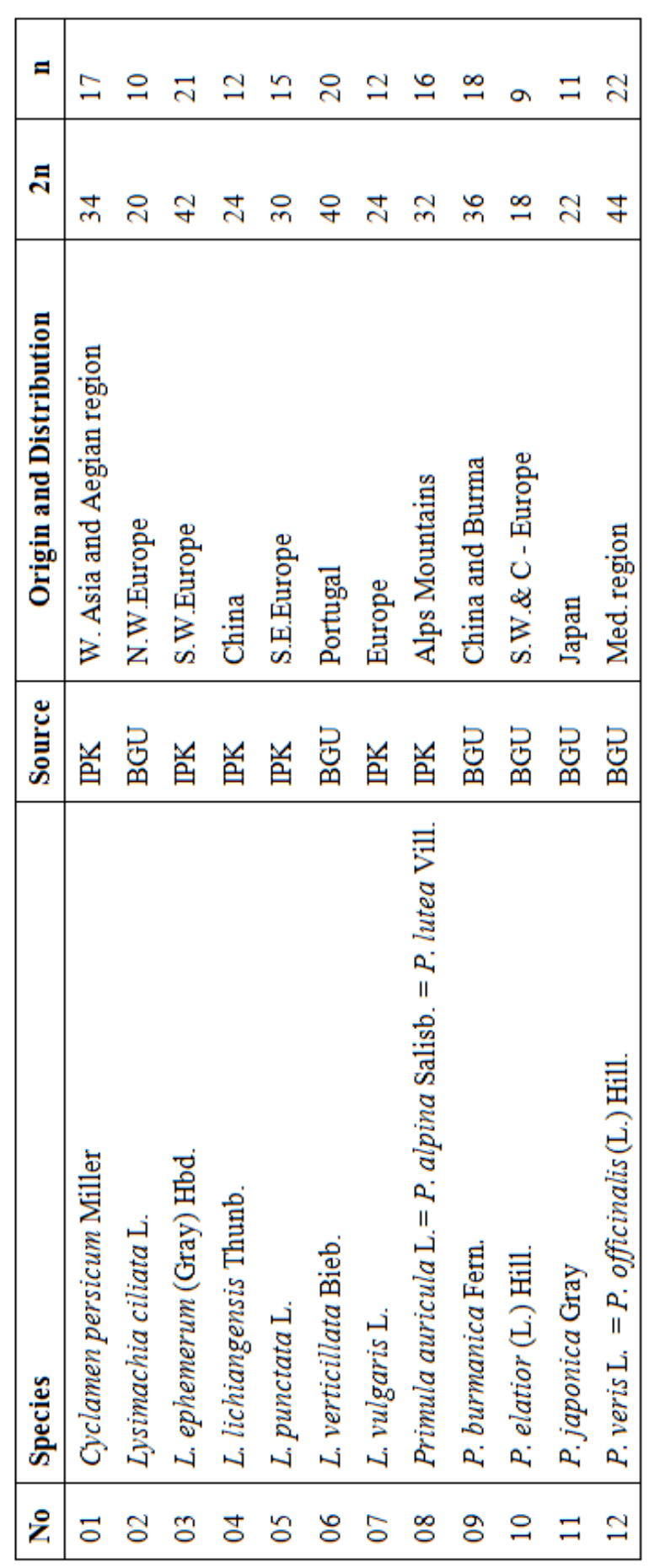

穿

๖

造

范

费

壱

葛

온

ఖ

.ִ

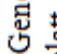

节吾

窟

㟧 可

总这

응

站弯

II

光

竞密

究

范

ต .

穿泀

.

药

ํํํ

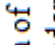

氙

of

]

矛

๓๐

总 
Table (2): The characters and their codes used in the present study for the numerical analyses

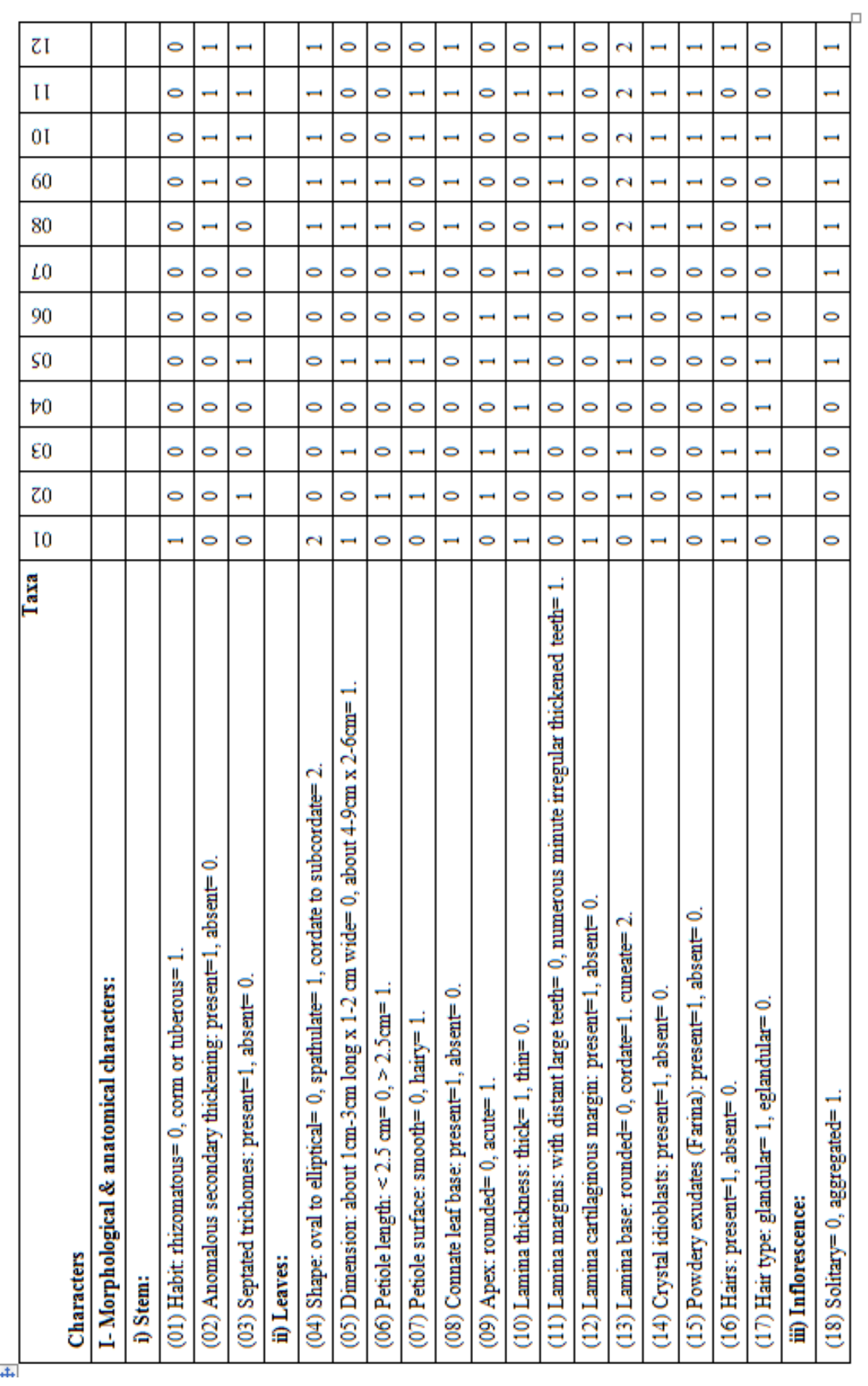


Table (2): Cont.

\begin{tabular}{|c|c|c|c|c|c|c|c|c|c|c|c|c|c|c|c|c|c|c|c|c|c|c|}
\hline ZI & $\rightarrow$ & $\rightarrow$ & $\rightarrow$ & $\rightarrow$ & $\rightarrow$ & $\rightarrow$ & $\rightarrow$ & & 0 & 0 & $\rightarrow$ & 0 & $\circ$ & $\rightarrow$ & $\rightarrow$ & $\rightarrow$ & 0 & 0 & $\circ$ & & $\mathrm{N}$ & 0 \\
\hline II & $\rightarrow$ & 0 & 0 & $\rightarrow$ & $\rightarrow$ & 0 & $\rightarrow$ & & $\rightarrow$ & 0 & $\rightarrow$ & $\rightarrow$ & 0 & 0 & $\rightarrow$ & $\rightarrow$ & 0 & 0 & $\circ$ & & $\mathrm{N}$ & 0 \\
\hline $0 \mathrm{I}$ & $\rightarrow$ & $\rightarrow$ & 0 & 0 & $\rightarrow$ & - & $\rightarrow$ & & 0 & 0 & $\rightarrow$ & 0 & 0 & 0 & $\rightarrow$ & $\rightarrow$ & 0 & 0 & $\circ$ & & $\mathrm{N}$ & 0 \\
\hline 60 & $\rightarrow$ & $\rightarrow$ & $\rightarrow$ & 0 & $\rightarrow$ & 0 & 0 & & 0 & $\rightarrow$ & $\rightarrow$ & $\rightarrow$ & $\rightarrow$ & $\rightarrow$ & $\rightarrow$ & $\rightarrow$ & 0 & 0 & 0 & & $\mathrm{~A}$ & 0 \\
\hline 80 & $\rightarrow$ & 0 & 0 & 0 & $\circ$ & $\rightarrow$ & $\rightarrow$ & & $\rightarrow$ & $\circ$ & $\rightarrow$ & $\circ$ & $\circ$ & $\rightarrow$ & $\rightarrow$ & $\rightarrow$ & 0 & 0 & $\circ$ & & $\mathrm{N}$ & 0 \\
\hline$\angle 0$ & a & $\rightarrow$ & $\rightarrow$ & $\rightarrow$ & $\rightarrow$ & 0 & 0 & & 0 & $\rightarrow$ & 0 & $\rightarrow$ & $\rightarrow$ & $\rightarrow$ & 0 & 0 & - & 0 & $\circ$ & & $\rightarrow$ & 0 \\
\hline 90 & $\mathrm{a}$ & 0 & 0 & 0 & 0 & 0 & 0 & & -1 & 0 & 0 & $\circ$ & $\circ$ & 0 & 0 & 0 & $\rightarrow$ & 0 & $\circ$ & & $\rightarrow$ & 0 \\
\hline ऽ0 & a & $\rightarrow$ & $\rightarrow$ & $\rightarrow$ & $\rightarrow$ & $\rightarrow$ & 0 & & 0 & 0 & 0 & 0 & 0 & 0 & 0 & 0 & - & 0 & 0 & & $\rightarrow$ & 0 \\
\hline to & a & $\rightarrow$ & $\circ$ & 0 & $\circ$ & $\circ$ & $\circ$ & & - & $\circ$ & $\circ$ & $\rightarrow$ & $\rightarrow$ & $\circ$ & $\circ$ & $\circ$ & $\rightarrow$ & 0 & $\circ$ & & $\rightarrow$ & 0 \\
\hline $\mathcal{E}_{0}$ & a & 0 & $\circ$ & 0 & $\rightarrow$ & - & $\circ$ & & 0 & 0 & $\circ$ & $\circ$ & $\rightarrow$ & $\rightarrow$ & 0 & $\circ$ & - & 0 & $\circ$ & & $\rightarrow$ & 0 \\
\hline 20 & a & 0 & 0 & $\rightarrow$ & 0 & 0 & 0 & & 0 & 0 & $\rightarrow$ & $\rightarrow$ & $\rightarrow$ & $\rightarrow$ & 0 & 0 & $\rightarrow$ & 0 & $\circ$ & & $\rightarrow$ & 0 \\
\hline I0 & $\circ$ & $\rightarrow$ & $\rightarrow$ & $\circ$ & $\circ$ & $\circ$ & 0 & & $\rightarrow$ & $\rightarrow$ & 0 & 0 & $\rightarrow$ & 0 & 0 & $\rightarrow$ & 0 & - & $\rightarrow$ & & 0 & $\rightarrow$ \\
\hline 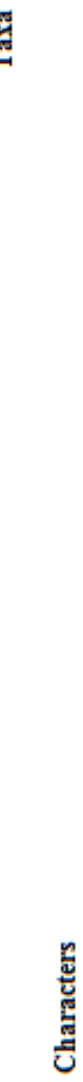 & 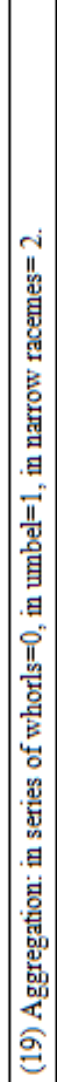 & 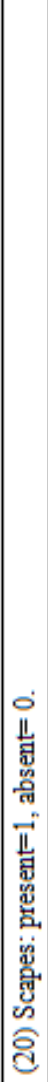 & 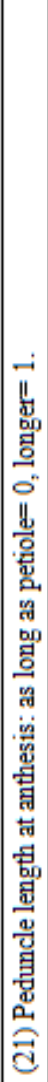 & 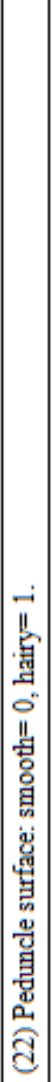 & 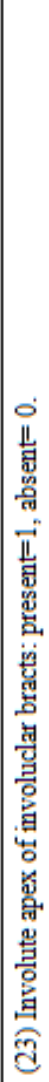 & 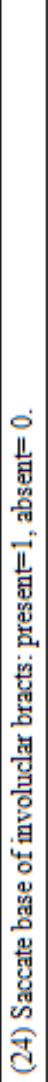 & 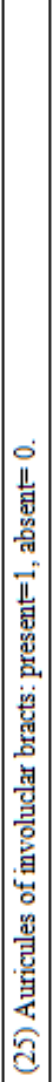 & $\begin{array}{l}\ddot{y} \\
\text { 总 } \\
\text { 总 }\end{array}$ & 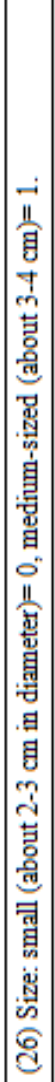 & 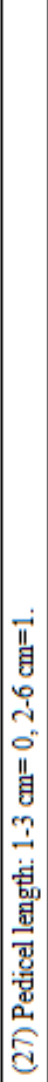 & 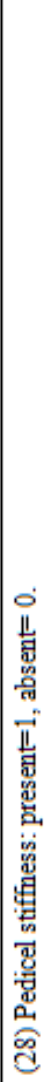 & 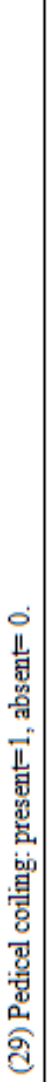 & 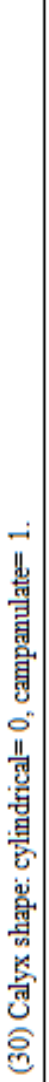 & 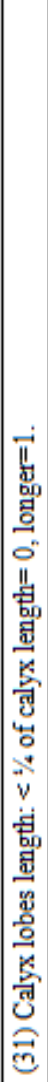 & 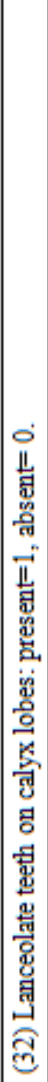 & 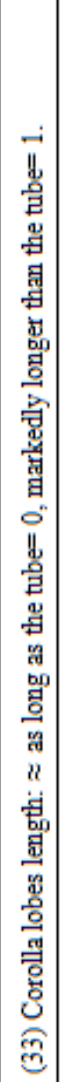 & 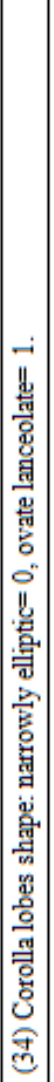 & 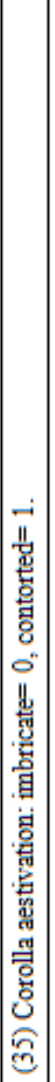 & 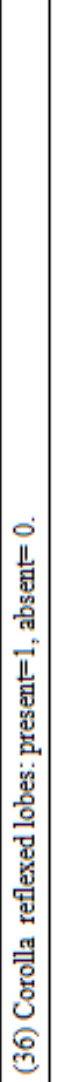 & 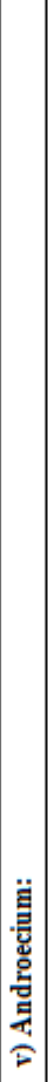 & 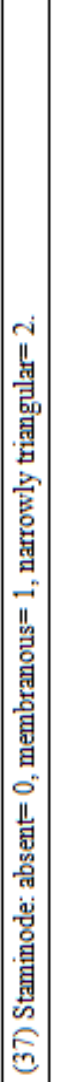 & 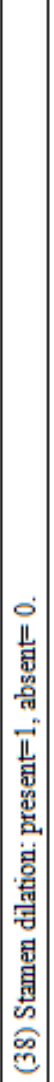 \\
\hline
\end{tabular}


Seed coat surface and seed protein criteria of Primulaceae

Table (2): Cont.

\begin{tabular}{|c|c|c|c|c|c|c|c|c|c|c|c|c|c|c|c|c|c|c|c|c|}
\hline $\mathrm{ZI}$ & - & 0 & - & $\circ$ & 0 & - & & $\rightarrow$ & $\circ$ & $\circ$ & $\rightarrow 0$ & 00 & 0 & & 0 & 0 & & - & - & \\
\hline II & $\rightarrow$ & 0 & - & 0 & -1 & 0 & & $\rightarrow$ & $\rightarrow$ & 0 & $\circ$. & 0 & 0 & & 0 & o & & - & -1 & \\
\hline $0 \mathrm{I}$ & $\rightarrow$ & 0 & 0 & 0 & 0 & - & & $\rightarrow$ & $\circ$ & $\circ$ & $\rightarrow 0$ & 00 & 0 & & $\circ$ & 0 & & - & - & \\
\hline 60 & - & 0 & - & $\circ$ & - & - & & $\rightarrow$ & - & 0 & 0. & -10 & - & & 0 & 0 & & - & -1 & \\
\hline 80 & - & 0 & 0 & $\circ$ & $\circ$ & 0 & & $\rightarrow$ & $\rightarrow$ & $\circ$ & $\rightarrow-$ & -10 & - & & $\circ$ & 0 & & - & - & \\
\hline$\angle 0$ & - & 0 & - & $\circ$ & - & 0 & & 0 & $\circ$ & $\circ$ & $\rightarrow 0$ & $0-$ & 0 & & - & 0 & & 0 & $-\rightarrow$ & \\
\hline 90 & - & - & - & $\circ$ & 0 & - & & $\circ$ & $\circ$ & $\circ$ & $\circ 0$ & $0-$ & 0 & & $\rightarrow$ & - & & 0 & - & \\
\hline$\varsigma_{0}$ & - & 0 & 0 & $\circ$ & - & 0 & & 0. & $\rightarrow$ & $\circ$ & $\rightarrow 0$ & $0-$ & - & & - & - & & 0 & 0 & \\
\hline to & $\rightarrow$ & - & - & $\circ$ & 0 & 0 & & 0. & $\circ$ & $\circ$ & $\circ-$ & $\rightarrow-$ & 0 & & - & 0 & & 0 & - & \\
\hline$\varepsilon 0$ & - & 0 & 0 & $\circ$ & - & - & & $\rightarrow$ & $\rightarrow$ & $\circ$ & $\rightarrow 0$ & $0-$ & 0 & & $\rightarrow$ & 0 & & 0 & 0 & \\
\hline 20 & - & 0 & 0 & $\circ$ & - & - & & $\circ$ & $\circ$ & $\circ$ & $\circ-$ & $\rightarrow-$ & 0 & & $\rightarrow$ & 0 & & 0 & - & \\
\hline 10 & $\circ$ & - & - & - & 0 & 0 & & $\sim$ & $\rightarrow$ & - & $\rightarrow 0$ & $0-$ & - & & $\sim$ & $\rightarrow$ & & - & 0 & \\
\hline & 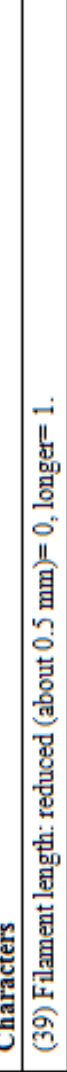 & 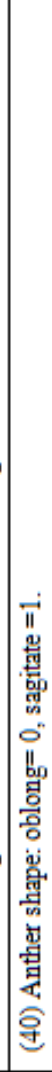 & 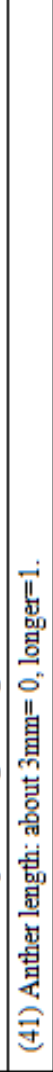 & 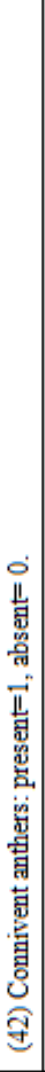 & 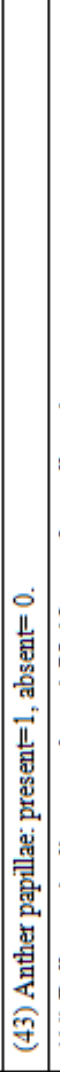 & 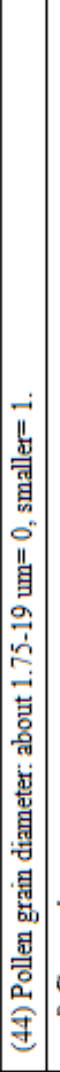 & $\begin{array}{l}\text { 首 } \\
\text { 恖 } \\
\text { 总 }\end{array}$ & 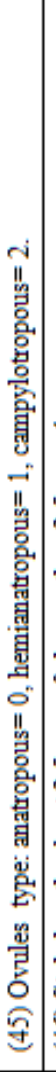 & 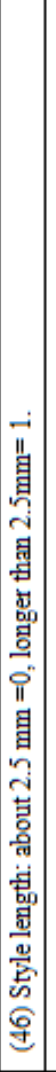 & 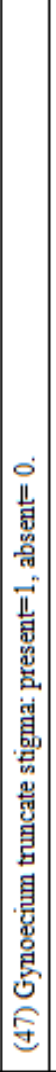 & \begin{tabular}{l|l} 
& \\
& \\
\end{tabular} & 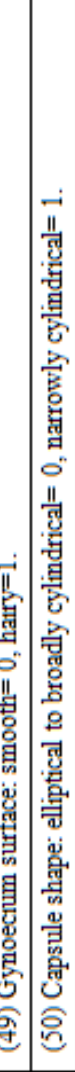 & 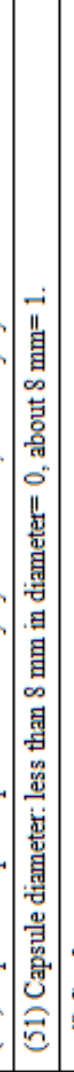 & 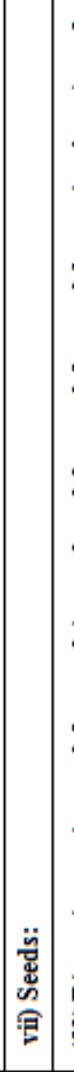 & 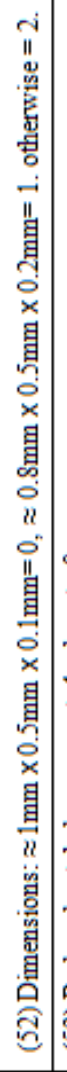 & 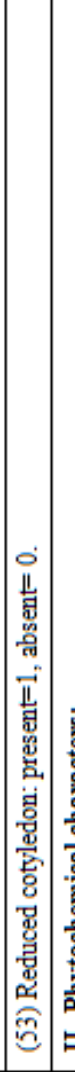 & 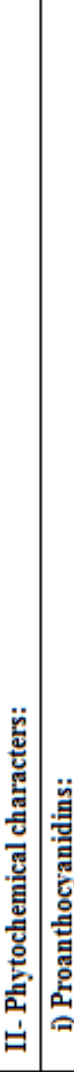 & 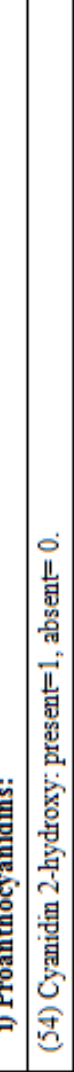 & 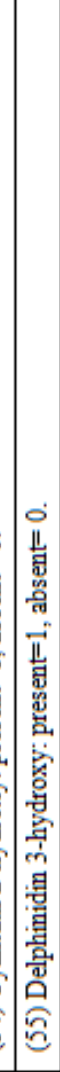 & 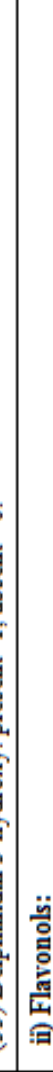 \\
\hline
\end{tabular}


Table (2): Cont.

\begin{tabular}{|c|c|c|c|c|c|c|c|c|c|c|c|c|c|c|c|c|c|c|c|c|c|c|}
\hline ZI & - & 0 & . & & & 0 & $\rightarrow$ & 0 & & $1-$ & -1. & $\rightarrow$ & -1 & $\rightarrow$ & - & - & $\rightarrow$ & & & 0 & - & $\circ$ \\
\hline II & $\rightarrow$ & 0 & - & & & 0 & $\rightarrow$ & 10 & & $c$ & c & . & 0 . & 0 . & $\rightarrow$ & - & $\rightarrow$ & & & 0 & 0 & $\rightarrow$ \\
\hline $0 \mathrm{I}$ & $\rightarrow$ & 。 & - & & & 。 & - & o & & & -1. & $\rightarrow$ & $\circ$. & $\rightarrow$ & 0 & 0 & $\rightarrow$ & & & n & 0 & A \\
\hline 60 & - & ㅇ & $\circ$ & & & ○ & - & . & & & $\circ$ & $\circ$. & 0 . & 0. & $\rightarrow$ & $\rightarrow$ & $\rightarrow$ & & & + & $\circ$ & $\mathrm{C}$ \\
\hline 80 & $\rightarrow$ & $\rightarrow$ & - & & & ○ & - & o & & & $-c$ & $\circ$. & -1 & 0. & 0 & 0 & $\rightarrow$ & & & 4 & 0 & ○ \\
\hline$\llcorner 0$ & $\rightarrow$ & 。 & - & & & 0 & ○ & - & & & 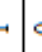 & $\circ$ & ०. & 0. & 0 & 0 & 0 & & & $\rightarrow$ & 0 & ○ \\
\hline 90 & $\rightarrow$ & o & o & & & 0 & ○ & - & & & -1 & 0 & 0 . & 0 . & 0 & 0 & 0 & & & 0 & 0 & - \\
\hline$\varsigma_{0}$ & ○ & $\rightarrow$ & o & & & - & o & - & & & 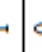 & $\circ$ & ०. & 0. & 0 & 0 & 0 & & & 0 & 0 & 。 \\
\hline t0 & $\rightarrow$ & 임 & - & & & ○ & ㅇ & - & & $c$ & $\circ$ & $\circ$. & $\circ$. & $\circ$ & $\circ$ & $\circ$ & $\circ$ & & & 0 & 0 & - \\
\hline$\varepsilon_{0}$ & $\rightarrow$ & 。 & - & & & 。 & 。 & - & & 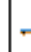 & $\rightarrow$ & $\circ$. & ०. & 0 . & 0 & 0 & 0 & & & 0 & 0 & 。 \\
\hline$z_{0}$ & - & 。 & $\circ$ & & & 0 & $\circ$ & - & & r & 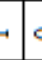 & 0. & 0 . & 0. & 0 & 0 & 0 & & & $m$ & 0 & - \\
\hline I0 & $\rightarrow$ & $\rightarrow$ & - & & & $\rightarrow$ & - & o & & - & -1. & $\rightarrow$. & -1 & -1 & 0 & 0 & 0 & & & a & 0 & ○ \\
\hline 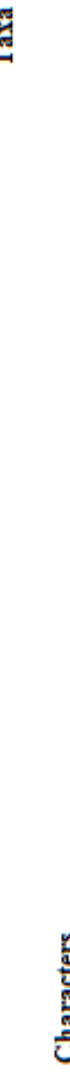 & 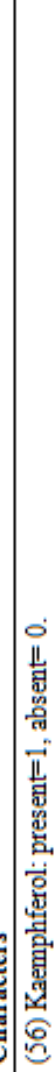 & 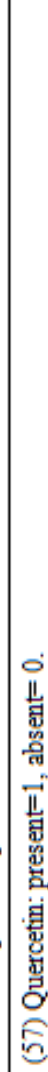 & 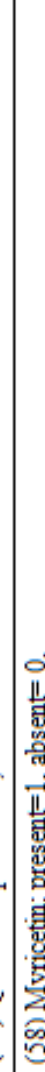 & $\begin{array}{l}2 \\
0 \\
0 \\
0 \\
0\end{array}$ & 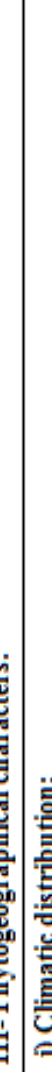 & 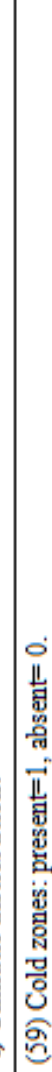 & 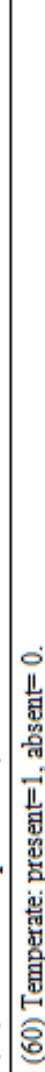 & 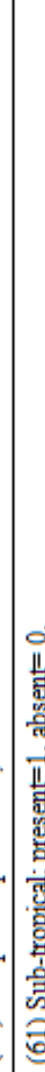 & 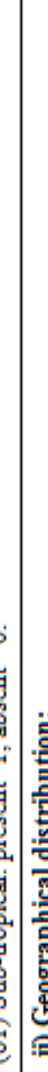 & iI & 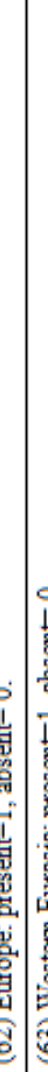 & 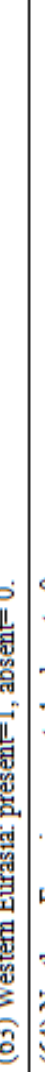 & 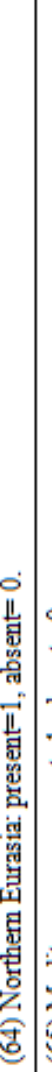 & 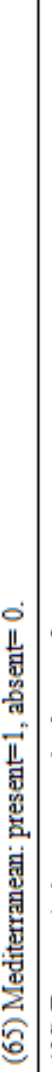 & 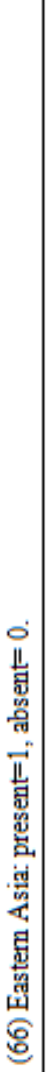 & 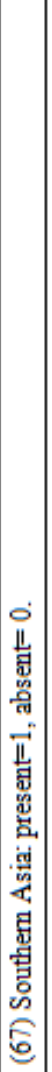 & 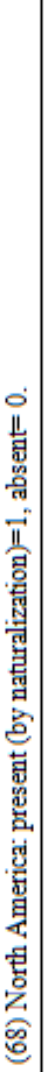 & 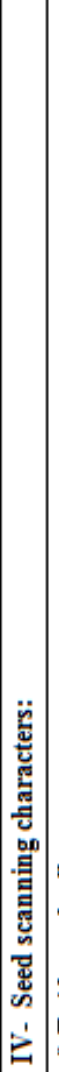 & 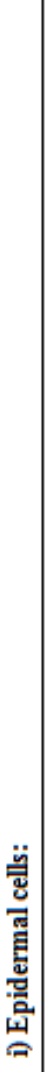 & 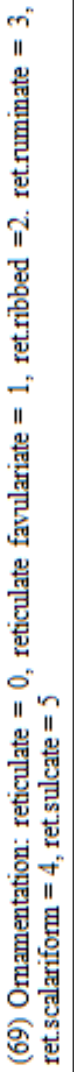 & 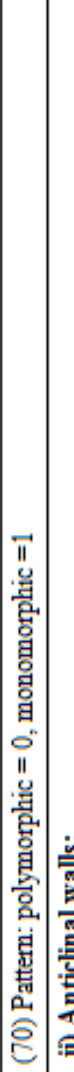 & 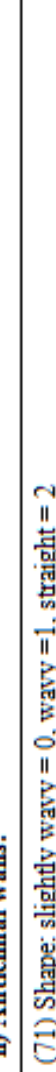 \\
\hline
\end{tabular}


Table (2): Cont.

\begin{tabular}{|c|c|c|c|c|c|c|c|c|c|c|c|c|c|c|c|c|c|c|c|c|c|c|}
\hline ZI & $\mathrm{s}$ & $\rightarrow$ & - & 0 & & -1 & 0 & $\rightarrow$ & & & $\circ$ & & 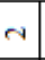 & & \begin{tabular}{l|c} 
& $c$ \\
\end{tabular} & 0. & \begin{tabular}{l|l}
- & 0 \\
\end{tabular} & \begin{tabular}{|l|l}
0 &. \\
\end{tabular} & \begin{tabular}{|l|l} 
&. \\
\end{tabular} & $\rightarrow$ & 0. & \\
\hline II & $\mathrm{a}$ & 0 & A & 0 & & - & $\rightarrow$ & 0 & & & 0 & & N & & 0 . & $\rightarrow-1$ & $\rightarrow$ & $\rightarrow$. & $-\rightarrow$ & $\rightarrow$ & 0 & $\circ$ \\
\hline $0 \mathrm{I}$ & $m$ & $\rightarrow$ & $\rightarrow$ & $\rightarrow$ & & 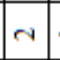 & $\rightarrow$ & $\rightarrow$ & & & 0 & & 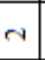 & & $\rightarrow$. & $\rightarrow$ & $\rightarrow$ & -1 & $\begin{array}{lll}. & . \\
\end{array}$ & $\rightarrow$ & $\circ$. & $\rightarrow$ \\
\hline 60 & 7 & $\rightarrow$ & $\mathrm{A}$ & $\rightarrow$ & & 0 & 0 & ० & & & $\circ$ & & ה & & 0. & -1. & $\rightarrow$ & -1 & $\begin{array}{lll}. & . \\
\end{array}$ & $\rightarrow$ & 0. & - \\
\hline 80 & + & $\rightarrow$ & 0 & 0 & & $\mathrm{~A}$ & $\sim$ & $\rightarrow$ & & & 0 & & $\mathrm{~N}$ & & 0. & $-a_{0}$ & \begin{tabular}{|l|l}
0 &. \\
\end{tabular} & $-\rightarrow$ & $\rightarrow$ & $\rightarrow$ & $\circ$. & - \\
\hline$\angle 0$ & $m$ & 4 & - & 。 & & 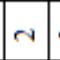 & 0 & 0 & & & 0 & & 0 & & $\rightarrow c$ & 0.0 & \begin{tabular}{l|l} 
& .1 \\
\end{tabular} & -1. & $\rightarrow$ & 0. & $\rightarrow$ & $\rightarrow$ \\
\hline 90 & A & 4 & $\mathrm{~A}$ & 0 & & - & $\mathrm{m}$ & - & & & 0 & & $\circ$ & & 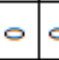 & \begin{tabular}{ll|l}
0 & 0 \\
\end{tabular} & \begin{tabular}{l|l} 
&. \\
\end{tabular} & -1. & $\rightarrow$ & 0. & -1. & - \\
\hline$\varsigma_{0}$ & A & 4 & 0 & 0 & & $\rightarrow$ & - & $\rightarrow$ & & & 0 & & 0 & & 0.0 & \begin{tabular}{l|c}
0 & 0 \\
\end{tabular} & \begin{tabular}{|l|l}
0 &. \\
\end{tabular} & $\rightarrow$ & $\rightarrow$ & 0. & $\rightarrow-$ & - \\
\hline t0 & $\rightarrow$ & ○ & $\mathrm{N}$ & - & & $\rightarrow$ & $\mathrm{m}$ & 0 & & & 0 & & 0 & & $\rightarrow c$ & 0.0 & \begin{tabular}{l|l} 
& .1 \\
\end{tabular} & -1. & $\rightarrow$ & 0. & $\rightarrow$ & $\rightarrow$ \\
\hline$\varepsilon_{0}$ & $\rightarrow$ & 4 & 0 & 0 & & $\mathrm{~A}$ & - & $\rightarrow$ & & & 0 & & $\circ$ & & 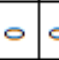 & \begin{tabular}{l|l}
0 & .1 \\
\end{tabular} & $\rightarrow$ & -1 & $\begin{array}{lll}\circ & 0 \\
\end{array}$ & 0. & -1. & - \\
\hline zo & A & 4 & $\mathrm{~A}$ & 0 & & 0 & 0 & 0 & & & 0 & & 0 & & $\rightarrow c$ & \begin{tabular}{l|c}
0 & 0 \\
\end{tabular} & \begin{tabular}{|l|l}
0 &. \\
\end{tabular} & $\rightarrow$ & \begin{tabular}{|l|l}
$\circ$ & 0 \\
\end{tabular} & 0. & $\rightarrow-$ & $\rightarrow$ \\
\hline 10 & + & $\rightarrow$ & $\mathrm{N}$ & 0 & & $\sim$ & $\sim$ & $\circ$ & & & $\rightarrow$ & & $\rightarrow$ & & $\rightarrow c 0$ & 0.0 & $\begin{array}{lll}0 & . \\
\end{array}$ & $\rightarrow$ & 0.0 & $\circ$. & $\rightarrow c$ & 0 \\
\hline & 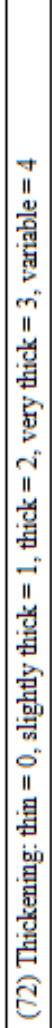 & 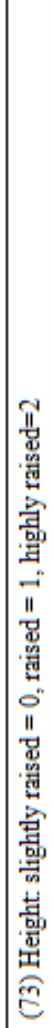 & 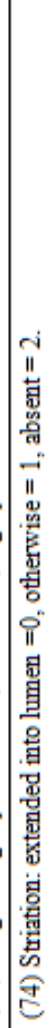 & 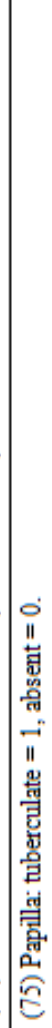 & 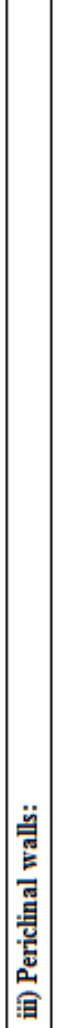 & 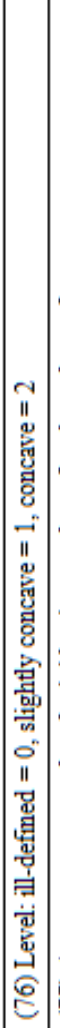 & 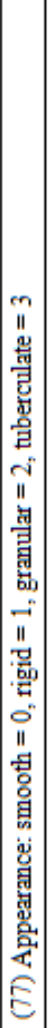 & 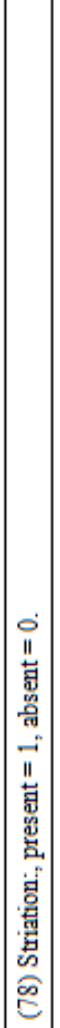 & 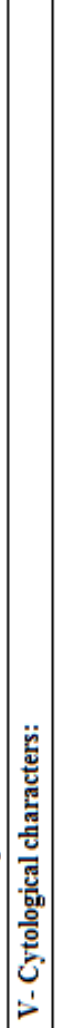 & 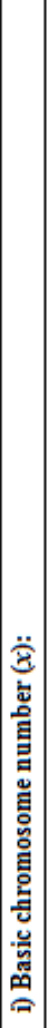 & 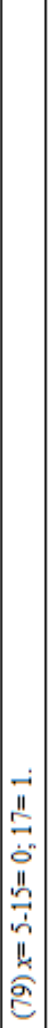 & 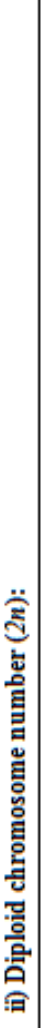 & 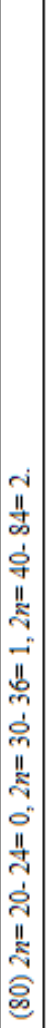 & 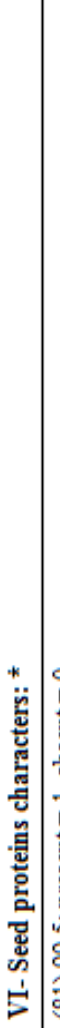 & 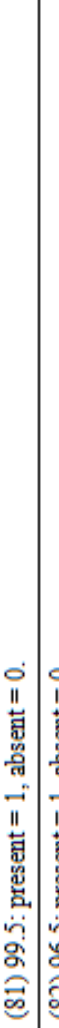 & 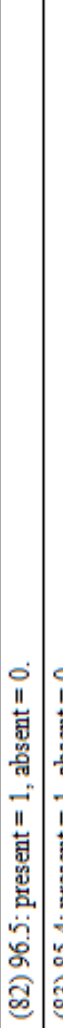 & 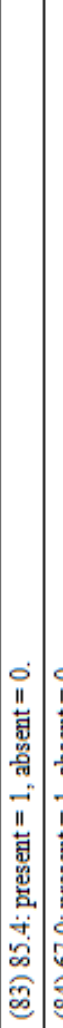 & 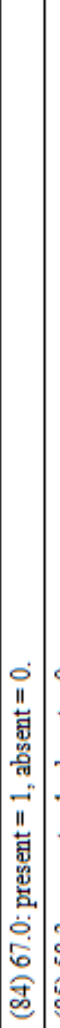 & 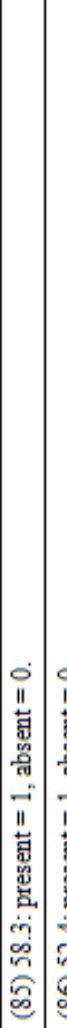 & 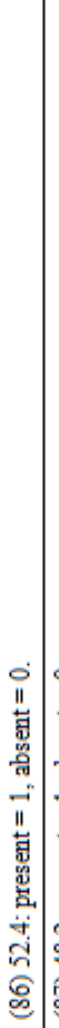 & 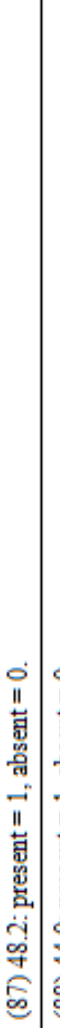 & \\
\hline
\end{tabular}


Table (2): Cont.

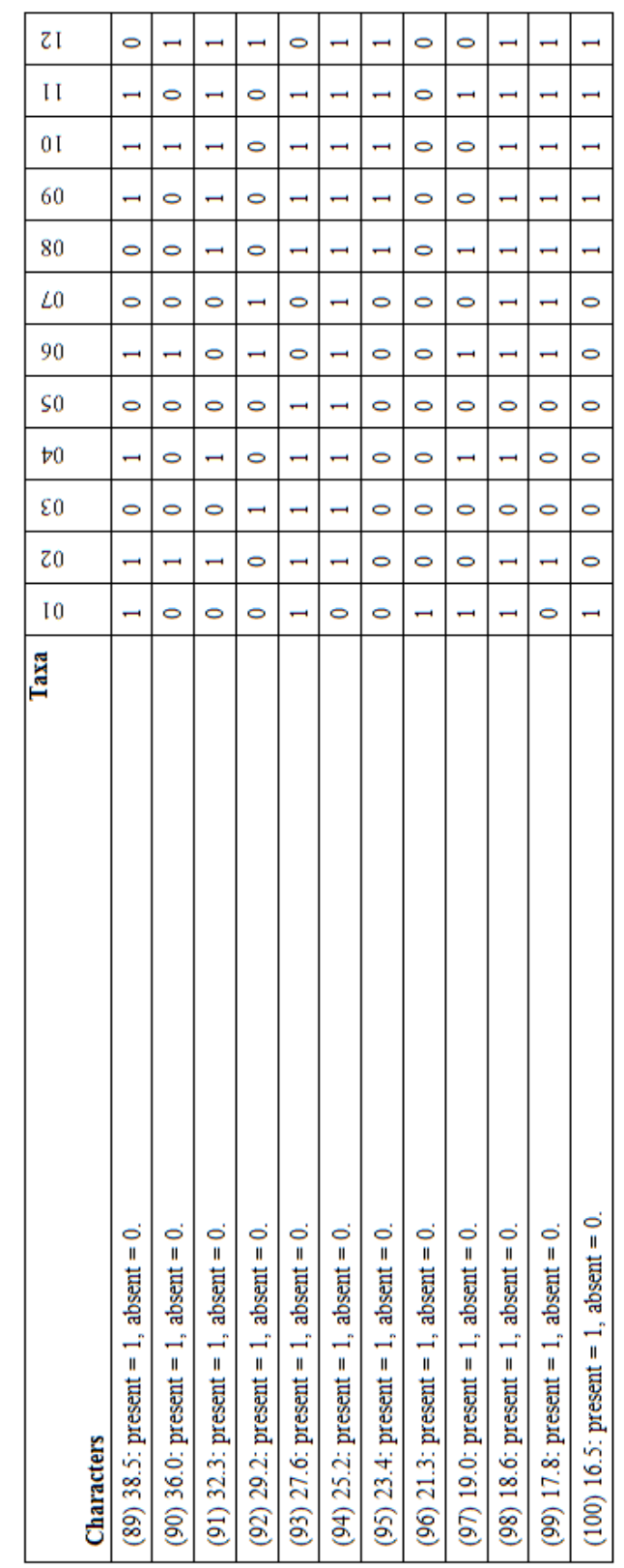

- The taxa studied are numbered as in table 1.

- Characters from 81-100 represents the molecular weights (in KD) of the recorded protein bands . the presence or absence of each band is coded 1 or 0 , respectively. 


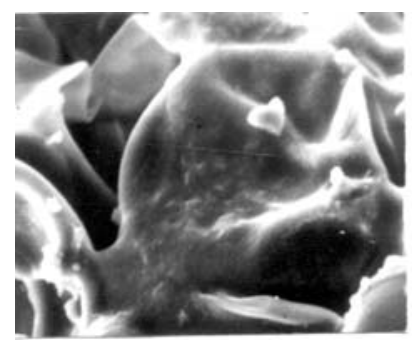

1

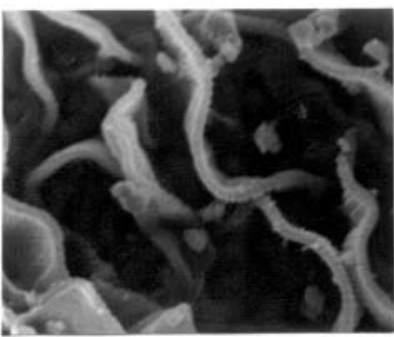

4

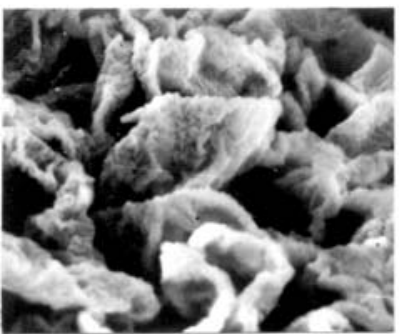

7

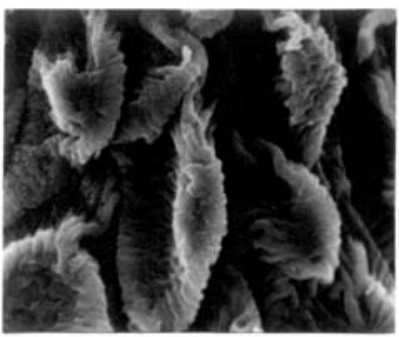

10

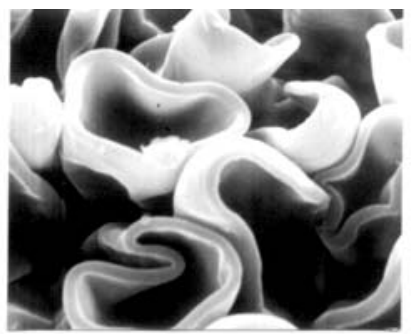

2

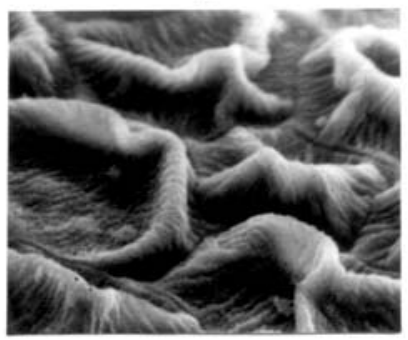

5

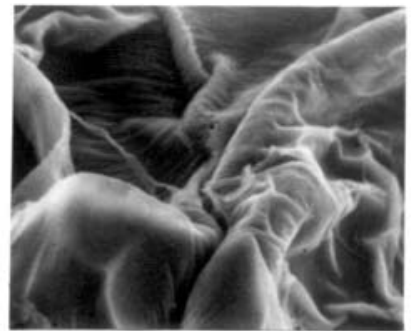

8

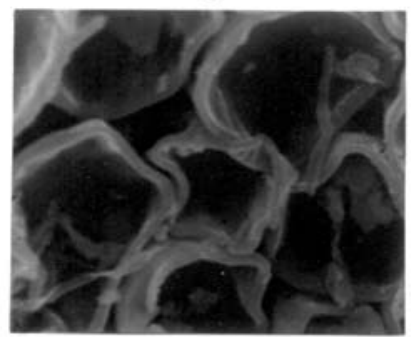

11

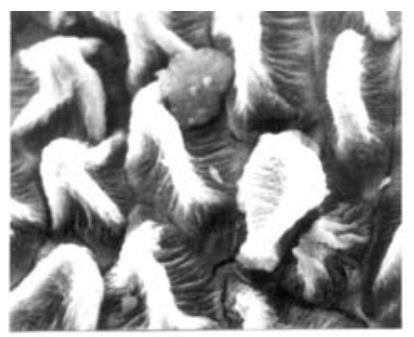

3

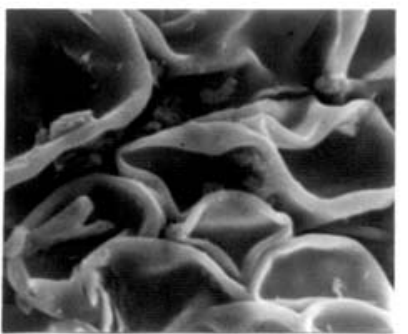

6

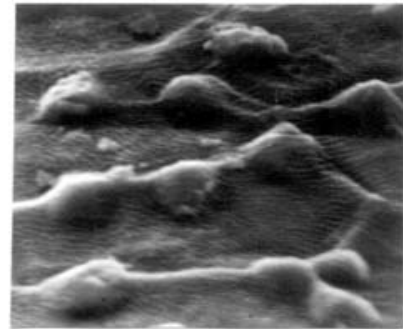

9

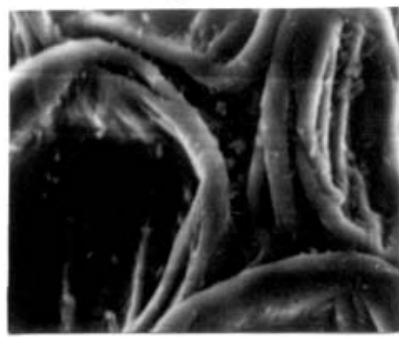

$1210 \mathrm{man}$

Fig. 1: Micrographs of the SEM patterns of the seed coat surface in each of the taxa studied.

1. Cyclamen persicum, 2. Lysimachia ciliata, 3. L. ephemerum , 4. L. lichiangensis, 5. L. punctata, 6. L. verticillata, 7. L. vulgaris, 8. Primula auricula, 9. P. burmanica, 10. P. elatior, 11. P. japonica, 12. P. veris. 


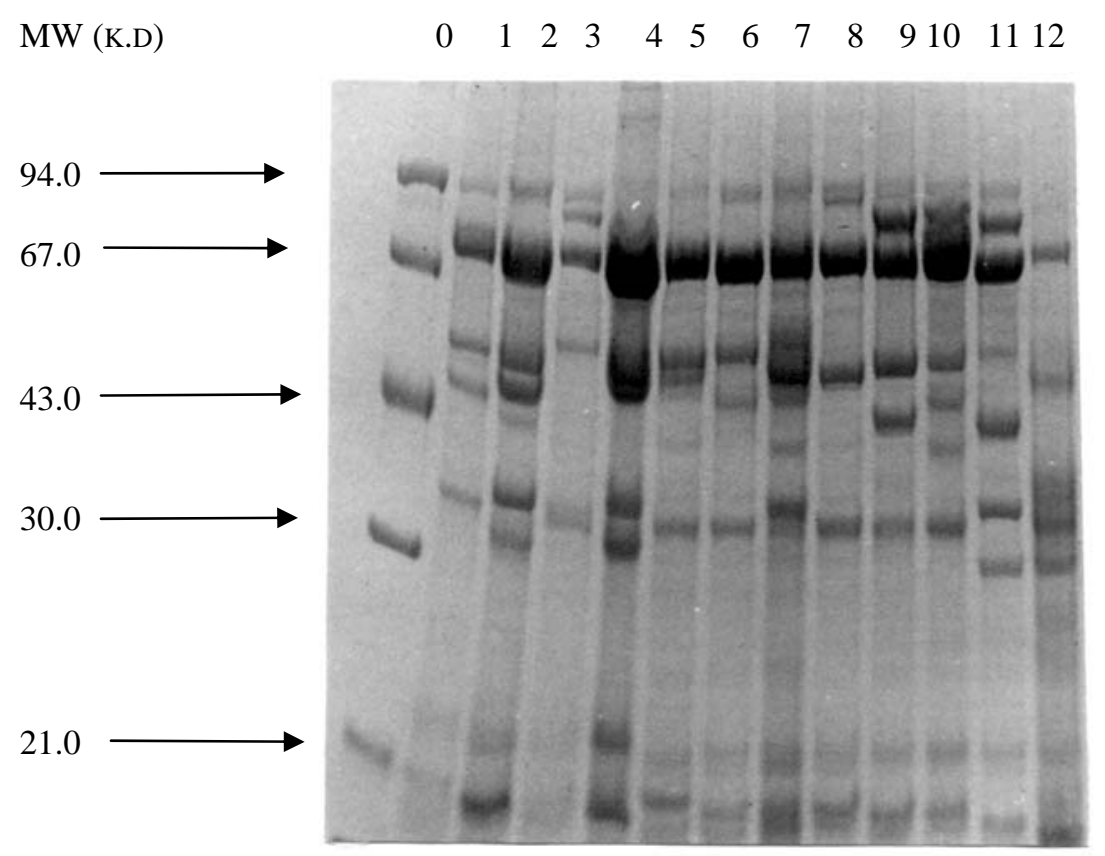

Fig. 2: Banding patterns of seed protein electrophoresis using SDS-PAGE technique. (0. Marker, 1. Cyclamen persicum, 2. Lysimachia ciliata, 3. L. ephemerum , 4. L. lichiangensis, 5. L. punctata, 6. L. verticillata, 7. L. vulgaris, 8. Primula auricula, 9. P. burmanica, 10. P. elatior, 11. P. japonica, 12. P. veris. 


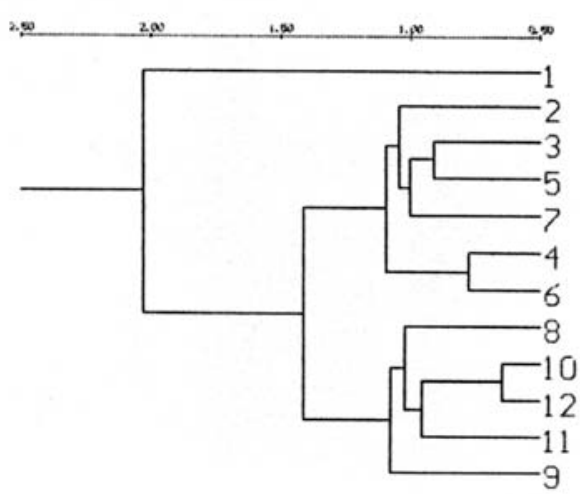

A

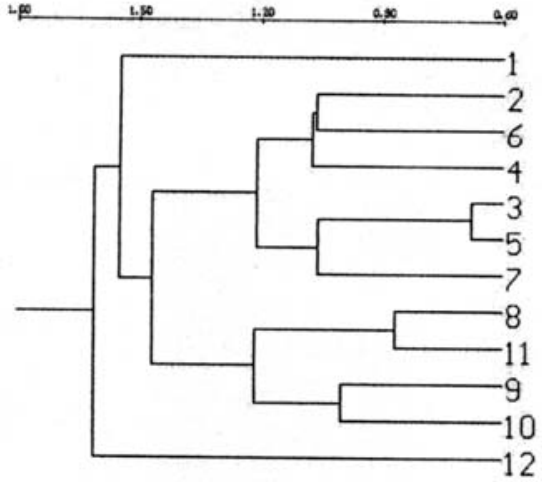

C

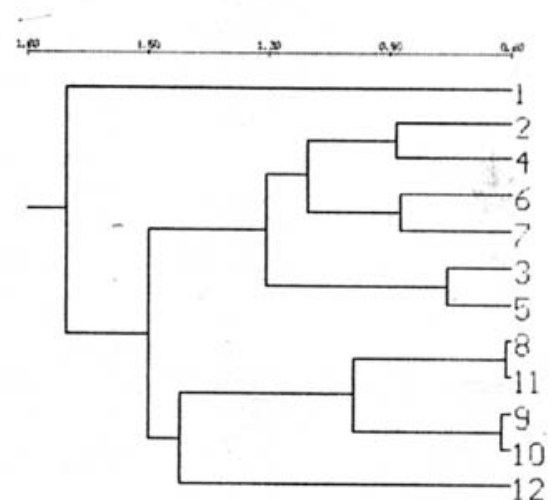

B

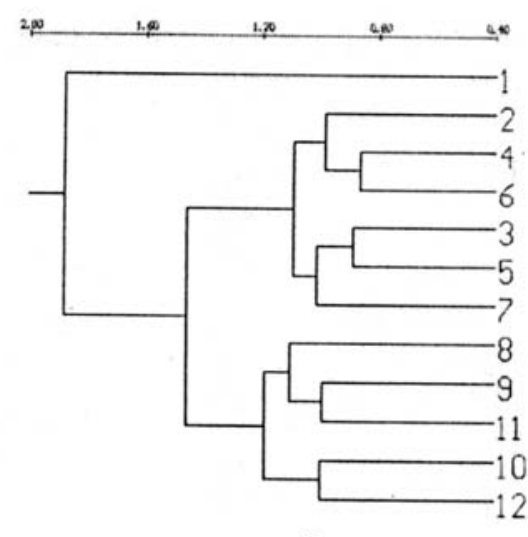

D

Fig. 3, A-D: Phenograms illustrating the relationships between the taxa studied; based on the character analyses using UPGMA clustering: A. Morphology, B. SDS-PAGE, C. Combination of SEM \& SDS-PAGE and D. All data combined. 
11. P japonica Gray Similar to Lysimachia verticillata except that the cell size is comparatively larger.

12. $\boldsymbol{P}$. veris L. Spermoderm reticulate, cells rounded, large in size and monomorphic. Anticlinal walls slightly wavy, raised and very thick. Each cell has a secondary wall thickening along the cell margin (superficially seen branching from the cell walls). Periclinal walls slightly concave and slightly striated.

Barthlott (1981) stressed on the significance of seed coat microsculpture in the characterization and delimitation of taxa at the sub familial level down to the sub generic level. Here, the SEM data for the studied taxa showed a considerable degree of similarity and even some overlap, suggesting the monophyly of the family; a fact that was realized by Anderberg \& Stahl (1995) and Judd et al. (1999) who stated that the monophyly of the Primulaceae s.l. is now strongly supported by morphological characters. However, the SEM data revealed a marked similarity between Cyclamen persicum (Cyclaminae) and Primula auricula (Primuleae). This result is in accordance with Anderberg (1994) who reported on the similarity of Cyclamen with certain genera of the Primuleae as Dodecatheon L. and Soldanella L. The first genus is a Pacific North American and was sometimes placed in the Cyclamineae by Pax, (1889) and Schwarz, (1955). The latter genus is native to the European Alps. Cyclamen also shares with Primula, the characteristic rosette form of growth ( Beckett, 1983).

Finally, the marked variations recorded between the studied taxa of Primula, and also the similarity between P. japonica (11) and both Lysimochia ciliata (2) and L. verticillata (6) suggest that Primula (37 sections and 400 species sensu Mabberley 1997) might prove to be paraphyletic (Fig. 1 and Table 2).

Investigation of the SDS-PAGE data (Fig. 2 and Table 2) showed that the studied Cyclamen species differ from the studied species of Lysimachia and Primula due to differences in the following criteria: presence of the protein band number 16 (Character No. 96) and absence of the protein band number 14 (Ch. No. 94) with MW of 25.2 and 21.3 KD, respectively. On the other hand Lysimachia and Primula are grouped together due to the presence of the protein bands numbered 6 (Ch. No. 86), 7 (Ch. No.87), 11 (Ch. No.91), 14 (Ch. No.94), 15 (Ch. No.95) and 20 (Ch. No.100) with MW of 52.4, 48.2, 32.3, 25.2, 23.4 \& $16.5 \mathrm{KD}$, respectively. The latter two taxa viz. Lysimachia and Primula are characterized from each other by the presence of the protein band number 7 (Ch. No. 87) with MW of $48.2 \mathrm{KD}$ and absence of the protein bands numbered 6 (Ch. No. 86) and 15 (Ch. No. 95) with MW of 52.4 \& 23.4 KD, respectively.

The combination of SDS-PAGE \& SEM data showed a relatively homogenous nature of the studied taxa of Lysimachia. However, Primula veris (12) was shown to be delimited from the remaining taxa at a distance level of 1.61. This result may give extra support to the paraphyly of Primula, (Table 2 \& Fig. 3-C).

The phenogram based on the combination of all data (Fig. 3, D) shows that Cyclamen persicum (1) is delimited from the other taxa at a distance of 1.86. The remaining eleven taxa are divided into two groups corresponding to the genera Lysimachia and Primula and are distinguished from each other at a distance of 1.46. The first group includes two subgroups that are distinguished from each other at a distance of 1.09. The first subgroup comprises Lysimachia ciliata (2), L. lichiangensis (4) and $L$. verticillata (6); while the second comprises L. ephemerum (3) and L. punctata (5). The 
second group is also composed of two subgroups. The first subgroup includes Primula auricula (8), $P$. burmanica (9) and $P$. japonica (11). The second subgroup includes $P$. elatior (10) and $P$. veris (12).

Nishino (1983) and Beyazoglu (1989) reached a similar conclusion based on a study of the corolla tube-formation and the anatomy of the root, the stem and the leaf. Morphologically, Cyclamen is unique as regards the suppressed development of one of its cotyledons, the contorted aestivation of the petals, the reflexed corolla lobes, the connivent anthers with well developed connectives, the increased growth of the subterranean tuberous hypocotyls and the absence of the septated trichomes common in the Primulaceae (Grey-Wilson, 1988). On the other hand, Primula is characterized from Lysimachia by the presence of the idioblasts, the powdery exudates (Farina) and the anomalous secondary thickening (Kelso, 1991; Anderberg, 1994).

However, these results are contradicting those of Kallersjo et al (2000), who after a cladistic analysis of certain morphological and molecular criteria in several families of the Ericales (sensu APG, 1998), transferred certain genera of the Primulaceae s.l. as Anagalis, Cyclamen and Lysimachia to the Myrsinaceae s.str. (excluding the Maesoideae), while retaining Primula in the Primulaceae s.str (containing the Primuleae only). The same view is also upheld by Anderberg et al. (2000).

Comparison of the phenogram illustrating the relationships between the taxa studied based on all combined data (Fig. 3, D) with those based on the different data sets (Fig. 3, A-C) revealed a frequent clustering and close relationships between each of the following species pairs: 1- Lysimachia lichiangensis (4) \& L. verticillata (6), this relationship was observed in all classifications produced except in those based on either SDS-PAGE of seed proteins alone or on the combination of SEM and SDS- PAGE data sets (Fig. 3 B \& C, respectively). 2- L. ephemerum (3) \& L. punctata (5), this relationship was observed in all classifications produced (Fig. 3: A-D). 3- Primula auricula (8) \& P. japonica (11) this relationship was observed in all classifications produced except in that based on morphology alone or that based on all characters (Fig. 3A - D). 4- P. elatior (10) $\& P$. veris (12) that was recorded in all classifications produced except those based on SDS-PAGE alone and in combination with SEM data (Fig. 3 B \& C).

The general consistency recorded in the present study between the macromorphological classification (Fig. 3, A) and that based on seed coat microsculpture and / or on seed protein electrophoresis (Fig. 3, B\&C) reveals that, the variations in SDSPAGE of seed protein profiles is compatible to some extent with the morphological variations exhibited in these taxa. Moreover, the results support the use of SEM of seed coat and seed protein electrophoretic criteria as valid tools in the taxonomy of the Primulaceae.

Although, the present study gives more support to the monophyly of the family as presented by Judd et al (1999), yet further work on cosmopolitan material of the Primulaceae is needed for a comprehensive conclusion.

\section{References}

Anderberg A.A. 1994. Phylogeny and subgeneric classification of Cyclamen L. (Primulaceae). Kew Bull. 49: 455 - 467.

\& Stahl B. 1995. Phylogenetic interrelationships in the order Primulales with special emphasis on the family circumscriptions. Can.J.Bot. 73: 1966 - 1730. 
Anderberg A.A. \& Kallersjo M. 1998. Phylogenetic relationships in the Primulales inferred from rbcL. sequence data. Pl. Syst. Evol. 211: 93 - 102. ..; Stahl B. \& Kallersjo M. 2000. Maesaceae, a new primuloid family in the order Ericales. s.l. Taxon. 49:183 - 187.

APG (Angiosperm phylogeny group), 1998. An ordinal classification for the families of flowering plants. Ann. Missouri Bot. Gard. 85: 531-553.

Badr A., Abou-El-Enain M.M. \& El-Shazly, H.H., 1998. Variation in seed protein electrophoretic pattern and species relationships in Sesbania. 6th Egypt. Bot. Conf. Cairo University (November 24-26, 1998) III:493-501.

, El-Shazly H.H. \& Abou-El-Enain M.M. 2000. Seed protein diversity and its implications on the relationships in the genus Lathyrus. 1st Int. Conf. Biol. Sci., Tanta University (May 7-8, 2000). In Press.

Bailey L.H. 1949. Manual of cultivated plants. Macmillan Publ. Company, New York. Pp. 776 $-785$.

.. \& Bailey E.Z. 1976. "Hortus Third. A concise Dictionary of Plants cultivated in the U.S. \& Canada". "Revised by the Staff of the L.H.Bailey Hortorium". Macmillan Publ. Co. New York. Pp. 690, 691, 907 - 912.

Barthlott W. 1981. Epidermal and seed surface characters of plants: Systematic applicability and some evolutionary aspects. Nord. J. Bot. 1: 345-355.

Beckett K.A. 1983. The concise Encyclopedia of Garden Plants. Orbis Ltd. London Pp. 109, $110,318-322$.

Bennett S. T. \& Grimshaw J. M. 1991. Cytological studies in Cyclamen subg. Cyclamen (Primulaceae). Pl. Sys. Evol. 176:135-143.

Beyazoglu O. 1989. Anatomical studies on some taxa of the Primulaceae in northeastern Anatolia (Turkey). Doga-Turk-Botanic-Dergisi 13: 1-16.

Boesewinkel F.D. \& Bouman F. 1984. Seed structure. In: B.M. Johri (Ed.), Embryology of Angiosperms. Springer-Verlag, Berlin, Heidelberg, New York and Tokyo, Pp. 567608.

Fedorov A.A. 1969. Chromosome numbers of flowering plants. V.L. Komarov Botanical Inst., Leningrad. Pp: 588 - 595.

Goldblatt P. 1981. Cytology and phylogeny of Leguminosae. In Polhill, R. M. \& Raven, P. H., (Eds.): Advances in legume systematics, Part 2. Royal Bot. Gard. , Kew, Pp. 427463.

1988. Index to plant chromosome numbers 1984-1985. Monographs in Systematic Botany, Missouri Botanical Garden. Saint Louis. Missouri, USA. 23.

Grey-Wilson P. 1988. The genus Cyclamen. Kew Magazine monographs. Christopher Helm publishers Ltd. Royal Botanic Gardens, Kew, UK, Pp. 47-144.

Harborne J.B. 1968. Comparative biochemistry of the flavonoids, VII. Correlations between flavonoid pigmentation and systematics in the family Primulaceae. Phytochemistry 7: $1215-1230$.

Jorgenson C. A., Sorenson T. H. \& Westergard M. 1958. The flowering plants of Greenland: a taxonomical and cytological survey. Biol. Meddle. Kongel. Danske Vidensk. Selsk. 9: $1-172$.

Judd W.S.; Campbell C.S.; Kellog E.A. \& Stevens P.F. 1999. Plant Systematics. A Phylogenetic Approach. Sinauer Associates. Inc. U.S.A. Pp. 349.

Kallersjo M., Bergqvist G. \& Anderberg A.A. 2000. Generic realignments in Primuloid Families of the Ericales s.l. (Angiosperms): A Phylogenetic analysis based on DNA sequences from three Chloroplast genes and morphology. Am.J.Bot. 87 (in Press). 
Kelso S. 1991. Taxonomy of Primula Sects. Aleuritia and Armerina in North America. Rhodora 93: 67-99.

Khalifa S.F., Badr A., Aboel-Atta A. I. \& Abou-El-Enain M. M. 1998. Electrophoretic studies of seed proteins and their impact on the taxonomic relationships in the Solanaceae. J. Union Arab. Biol. 5:171-180.

Ladizinsky G. \& Hymowitz T. 1979. Seed protein electrophoresis in taxonomic and evolutionary studies. Theor. App. Genet. 54:145-151.

Laemmeli U. 1970. Cleavage of structural proteins during the assembly of the head of bacteriophage T4. Nature 227: 680-685.

Ludi W. 1927. Primulaceae. In: Hegi, G. (Ed.): Illustrierte Flora von Mitteleuropa 5 (3). Munchen. Carl Hanser.

Mabberley D.J. 1997. The plant book: A portable dictionary of the vascular plants, $2^{\text {nd }}$ Ed., Cambridge University Press, New York, Pp. 583- 584.

Nishino E. 1983. Corolla tube-formation in the Primulaceae and Ericales. Botanical Magazine. 96: 319-342.

Pax F.A. 1889. Primulaceae. In: Engler, A. \& Prantl, K. (eds). Die Naturlichen Pflanzenlamilien 4: 98 - 116. Engelmann, Leipzig.

\& Knuth R. 1905. Primulaceae. In: H. G. A. Engler (Ed.), Das Pflanzenreich Family IV. Wilhelm Engelmann, Berlin, Pp.1-386.

Pobedimova E.G. 1952: Primulaceae. In Shishkin, B. K. \& E.G. Bobrov (Eds.). Flora USSR 18. Akademii Nauk USSR, Moscow and Leningrad, Pp.108-292.

Porsild A.E. \& Cody W.J. 1980. Vascular plants of continental northwest territories. National Museums of Canada, Ottawa.

Rohlf F.J. 1993. NTSYS-pc, Numerical taxonomy and multivariate analysis system. Exeter Comp., New York.

Sarkar A.K. 1973. Cytotaxonomic studies on some members of Primulaceae from the Eastern Himalayas. Pro. Indian Sci. Congr. Assoc. 60: 316- 324. 1988. Primulaceae, its evolution and assessment in status as judged through cytotaxonomy. Fed. Repert. 99: 113-132.

Schwarz O. 1955. Systematische Monographie der Gattung Cyclamen. L. l. Feddes. Repert. 58: $234-283$.

. \& Lepper L. 1964. Kritische Revision der Gattung Cyclamen L. In Schwarz 0., Systematische Monographie der Gattung Cyclamen L. 2. Feddes. Repert. 69: 7992.

Spanowsky W. 1962. Die Bedeutung der pollenmorphologie fur die taxonomie der Primulaceae-Primuloideae. Fed. Repert. 65: 149-215.

Stearn, W.T. 1992. Botanical Latin, 4th edition. Timber Press, Portland, Oregon, Pp. 489-491.

Valentine, D.H.1972. Primulaceae in T.G.Tutin et al (editors), Flora Europaea Vol (3). Cambridge University Press. Pp. $14-29$.

Watson L. \& Dallwitz M. J. 1999. The Families of Flowering Plants, Descriptions, Illustrations, Identification, and Information Retrieval. CSIRO Publications, Melbourne. Pp. 248-269.

Watt J.M. \& Breyer-Brandwijk M.G. 1962. The Medicinal and Poisonous Plants of Southern and Eastern Africa. 2nd ed.. E. \& S. Livingstone Ltd., Edinburgh.

Yurtsev B.A., Petrovesky V.V., Korobkov A.A., Koroleva T.M. \& Razhiven V. Y. 1979. A review of the geographic distribution of vascular plants of the Chukotka tundra (in Russian). Byull. Moskovsk. Obsc. Isp. Prir. Otd. Biol. 84: 7- 83, 111-121. 